\title{
Correction to: Rapid development of HIV elite control in a patient with acute infection
}

Deirdre Morley ${ }^{1 *}$, John S. Lambert ${ }^{1,6}$, Louise E. Hogan², Cillian De Gascun³, Niamh Redmond ${ }^{4}$, Rachel L. Rutishauser ${ }^{2}$, Cassandra Thanh ${ }^{2}$, Erica A. Gibson ${ }^{2}$, Kristen Hobbs ${ }^{2}$, Sonia Bakkour ${ }^{5,7}$, Michael P. Busch ${ }^{5,7}$, Jeremy Farrell ${ }^{1}$, Padraig McGetrick' and Timothy J. Henrich ${ }^{2}$

\section{Correction to: BMC Infect Dis}

$$
\text { https://doi.org/10.1186/s12879-019-4374-8 }
$$

After publication of the original article [1], we were notified that a column needed to be removed from Table 1.

The correct version can be found below:

Furthermore, the words "Hiv specific antibody levels and" should be deleted from the 'Case presentation' section in the Abstract.

The original article has been corrected.

The publisher apologies for the inconvenience.

\begin{abstract}
Author details
${ }^{1}$ Mater Misericordae University Hospital, Eccles Street, Dublin 7, Ireland. ${ }^{2}$ Department of Medicine, University of California San Francisco Division of Experimental Medicine, 1001 Potrero Avenue, San Francisco, CA 94110, USA. ${ }^{3}$ National Virus Reference Laboratory, University College Dublin, Dublin, Ireland. ${ }^{4}$ UCD Clinical Research Centre, Dublin, Ireland. ${ }^{5}$ Vitalant Research Institute, 270 Masonic Ave, San Francisco, CA 94118, USA. ${ }^{6}$ University College Dublin School of Medicine, Dublin, Ireland. ${ }^{7}$ Department of Laboratory

Medicine, University of California, San Francisco, CA, USA.
\end{abstract}

Published online: 22 October 2019

\section{Reference}

1. Morley, et al. BMC Infect Dis. 2019;19:815. https//doi.org/10.1186/s12879-019-4374-8.

\footnotetext{
* Correspondence: deemorley@gmail.com

${ }^{1}$ Mater Misericordae University Hospital, Eccles Street, Dublin 7, Ireland

Full list of author information is available at the end of the article
} 
Table 1 Results of HIV molecular testing, Antigen/Antibody screening assay, and CD4 count from time first presentation (June 2014) to initiation of ARV therapy

\begin{tabular}{|c|c|c|c|c|c|c|c|}
\hline \multirow[t]{2}{*}{ Time } & \multicolumn{2}{|c|}{$\begin{array}{l}\text { Viral Load } \\
\text { (RNA copies/ml) }\end{array}$} & \multicolumn{3}{|c|}{$\begin{array}{l}\text { Forth Generation Antigen/Antibody HIV Test } \\
\text { (s/co -relative quantity of HIV Ab) }\end{array}$} & \multirow{2}{*}{$\begin{array}{l}\text { Confirmatory Test } \\
\text { INNO-LIA }\end{array}$} & \multirow{2}{*}{$\begin{array}{l}\text { CD4+ T } \\
\text { Cells/uL } \\
(\%)\end{array}$} \\
\hline & Viral Load & SCA & $\overline{\mathrm{ARCHITECT}}(\mathrm{S} / \mathrm{CO})$ & VIDAS (S/Co) & GS & & \\
\hline Jun 2014 & $71550^{\mathrm{a}}$ & - & $l^{b}$ & NEG & NEG & - & - \\
\hline Oct 2014 & $<200$ & - & $11.1^{\mathrm{c}}$ & $13.72^{c}$ & - & gp41 (3+), p31 (1+), p24 (3+), p17 (1+) c & - \\
\hline Nov 2014 & $<40$ & - & - & - & - & - & $616(45 \%)$ \\
\hline Dec 2014 & - & - & - & - & - & - & - \\
\hline Oct 2015 & $<40$ & - & - & - & - & - & $459(46 \%)$ \\
\hline Apr 2016 & $<40$ & - & - & - & - & - & $558(40 \%)$ \\
\hline Sep 2016 & - & $0.84^{d}$ & - & - & - & - & - \\
\hline \multicolumn{8}{|c|}{ Antiretroviral therapy commenced September $2016^{d}$} \\
\hline Oct 16 & - & - & - & - & - & - & 585 (47\%) \\
\hline
\end{tabular}

Fourth Generation HIV Antigen/Antibody test: ARCHITECT ${ }^{\oplus}$ Abbott; VIDAS ${ }^{\oplus}$ BioMerieux; GS = GeneScreen ${ }^{\oplus}$ Bio-Rad, INNO-LIA, Fujirebio ${ }^{\circ}$

SCA Single copy assay, S/Co Signal/cutoff

${ }^{\text {a }}$ Retrospective molecular test on stored sample from June 2014

${ }^{b}$ Read as equivocal value at time of testing

c Positive test

${ }^{d}$ ARV commenced on clinical grounds-patient presented with furunculosis 